\title{
On-line Detection and Classification of Rotor and Load Defects in PMSMs based on Hall Sensor Measurements
}

\author{
Yonghyun Park ${ }^{*}$, Chanseung Yang*, Sang Bin Lee*, Dong-Myung Lee ${ }^{* *}$, Daniel Fernandez ${ }^{* * *}$, David \\ Reigosa $^{* * *}$, and Fernando Briz ${ }^{* * *}$ \\ *Department of Electrical Engineering, \\ Korea University, Seoul, Korea \\ Email: sangbinlee@korea.ac.kr
${ }^{* *}$ School of Electronic and Electrical Engineering,
Hongik University, Seoul, Korea \\ ${ }^{* * *}$ Department of Electrical Engineering, \\ University of Oviedo, Oviedo, Spain
}

\begin{abstract}
On-line monitoring and diagnostics of permanent magnet synchronous motors (PMSM) is becoming important with the increasing demand in PMSM applications. Most of the research effort focuses on motor current signature analysis (MCSA) as it can provide remote, on-line monitoring at low cost. However, all types of defects that produce asymmetries in the PMSM rotor or load produce identical rotor rotational frequency components. This is a serious limitation when applying MCSA since it can produce false indications and degrade the sensitivity of fault detection. The requirement of complex time-frequency analysis techniques is another limitation of MCSA for applications with speed variations. In this paper, the feasibility of using analog Hall sensor signals to complement MCSA for detection and classification of rotor and load related defects is investigated. It is shown that Hall sensors installed in machines for initial rotor position estimation can be used with minimal hardware modifications, to detect and classify signatures produced by the rotor and load for cases where MCSA fails, even during transient conditions. Experimental testing performed on an IPMSM under eccentricity, local demagnetization, and load unbalance conditions show that the reliability and sensitivity of fault detection in PMSM systems can be improved at low added cost.
\end{abstract}

Index Terms-Axial Leakage Flux, Condition Monitoring, Fault Classification, Hall Effect Sensor, Load Unbalance, PM Demagnetization, PM Synchronous Motor, Rotor Eccentricity.

\section{INTRODUCTION}

Permanent magnet synchronous motors (PMSM) are replacing induction motors in many applications due to the benefits in energy efficiency and power density. With the increasing demand for high efficiency and precision applications, the quantity and power rating of PMSM drives are increasing. PMSM drive system components are prone to degradation due to manufacturing defects and electrical, mechanical, thermal, and environmental operating stresses. Defects in the motor and load such as rotor eccentricity, local PM demagnetization, load unbalance, misalignment, etc that produce mechanical asymmetry result in increased vibration. This results in degradation in system performance and accelerated wear in insulation, bearings, and other mechanical components that can lead to failure. Since there are many safety- and reliabilitycritical applications where PMSM drive system failures must be prevented, condition monitoring and diagnostics of PMSM drive systems has recently received a lot of attention [1]-[11].
Conventional offline tests such as visual inspection, dial test indicator, feeler gauge, and gaussmeter scanning tests can be applied for detecting rotor eccentricity and demagnetization [1]. There are also many conventional off-line techniques for checking rotating asymmetry such as unbalance and alignment that have been proven to work over the years [12]-[13]. Although these off-line tests can provide reliable assessment of asymmetry in the rotor and load of PMSM drive systems, they cannot be applied frequently, as they require full or partial motor disassembly, and/or specialized test equipment or setup.

To overcome the limitations of off-line testing, on-line monitoring for detecting rotating asymmetry produced by rotor eccentricity, local PM demagnetization, load unbalance, and misalignment have been studied in [2]-[11]. In [2]-[3], modelbased methods for detecting local demagnetization have been proposed; however, model based estimation is known to be influenced by non-ideal modeling errors and parameter variation. On-line detection of synchronous motor rotor faults based on vibration, shaft voltage, or flux spectrum analysis has been investigated in [4]-[6]. The rotor rotational speed frequency components, $f_{r}$, and their fundamental frequency, $f_{s}$, sidebands, $f_{\text {fault, }}$ given by

$$
\begin{gathered}
f_{r}=k \cdot f_{s} / p, \\
f_{\text {fault }}=f_{s} \pm f_{r}=(1 \pm k / p) f_{s},
\end{gathered}
$$

are monitored for fault detection, where $p$ is the number of pole pairs, and $k$ is an integer. Vibration spectrum analysis is known to be effective for detecting load unbalance, misalignment, and other mechanical defects in rotating systems, and has been verified to work in the field over a long period of time. The main limitation of shaft voltage, flux, or vibration monitoring is the additional cost and physical access required for retrofitting additional sensors on the surface of the motor.

Electrical and mechanical faults or imperfections that produce rotating asymmetries in the motor or load can also be detected with motor current signature analysis (MCSA) by monitoring the $f_{\text {fault }}$ component in the current spectrum. In [2][3], [7]-[11], it is shown that motor (rotor eccentricity, local PM demagnetization), coupling (gear/belt pulley faults), and load (load unbalance, misalignment, etc) defects can be detected with MCSA. Most of the recent studies focus on MCSA since it can provide remote, online monitoring with current measurements available in the drive. Despite the advantages, it was shown in [7]-[8] that the detectability of the fault depends on the winding 
configuration and motor topology. In addition, MCSA cannot be applied to PMSMs operating under non-stationary conditions; therefore, a computationally intensive time-frequency analysis technique is required for applications with speed and/or load variation [9]-[11]. One of the main limitations of MCSA comes from the fact that all rotating asymmetries that produce a once per revolution component, $f_{r}$, induce identical $f_{\text {fault }}$ sideband frequency components in the current, and there is no practical means of separating them [2]. This is a serious problem since multiple electrical/mechanical rotating asymmetries overlap at the same frequency in the current spectrum, and interact to add or cancel, as will be demonstrated in this paper. This can result in a costly false positive or negative indication, and degradation in the sensitivity of fault detection.

A literature review of PMSM drive system fault detection shows that conventional spectrum analysis based detection has many limitations in terms of detectability, reliability, and sensitivity. In this paper, the feasibility of utilizing the analog Hall sensors installed in PMSMs for initial position estimation, for the purpose of identifying the cause of rotating asymmetry is evaluated. An experimental study on an interior PMSM (IPMSM) under eccentricity, local PM demagnetization, and load unbalance conditions show that the Hall sensor output can be used in conjunction with MCSA to detect and classify PMSM rotor and load defects. It is also shown that the proposed method can be applied independent of speed variations to improve the sensitivity and reliability of fault detection with minimal hardware modification.

\section{ANALysis OF HALl SENSOR MEASUREMENTS UNDER PMSM ROTOR AND LOAD DEFECTS}

Since the absolute position of the rotor is required for control of PMSMs, many PMSM systems are equipped with Hall sensors for obtaining the rotor position. For PMSMs controlled with incremental encoders, Hall sensors are required for obtaining the initial position of the rotor. The output signals provided by Hall sensors are digitized in many PMSM drives for simplifying hardware and for noise immunity, as shown in Fig. 1. There are also commercial PMSM drives where the analog signal of the Hall sensors are used without digitization [14]-[15]. It has recently been shown that there are many benefits of using analog Hall sensor signals such as improvement in control performance [16]-[17] and monitoring of PM temperature [18]. It was also shown that airgap eccentricity and local demagnetization in the PMs can be detected with high sensitivity [19]-[21]. This is possible since Hall sensors are installed inside the motor in the space between the rotor and end shield to obtain the rotor position by measuring the leakage flux directly from the rotor as the PM passes the sensors. If the PMSM system is equipped with built-in analog Hall sensors, installation of additional sensors or hardware is not required for implementation of the proposed method. For cases where the Hall sensor measurements are digitized, modification of the system is required, as studied in [19]-[20].

\section{A. Rotor Eccentricity and Local PM Demagnetization}

The reference tangential $(x)$, radial $(y)$, and axial $(z)$ location and direction used to describe the location of the Hall sensor and the direction PM leakage flux measured are shown in Fig. 2. This is a 3 dimensional (3D) finite element (FE) model of a 7.5
kW, 6 pole IPMSM used for performing simulations under rotor eccentricity and local demagnetization faults. In this paper, the PM leakage flux in the axial ( $z$ ) direction is used with the Hall sensor located at $x=0$, and at nonzero $y$ and $z$ positions several millimeters from the geometric center of the PM, as shown in Fig. 2(b) $(y=4 \mathrm{~mm}, \mathrm{z}=5 \mathrm{~mm}$ for the Fig. 2 FE model). The Hall sensor flux density patterns in the $x-, y$-, and z-axis directions as a function of rotor flux angle are shown in Fig. 3(a). It was observed in previous studies that eccentricity and local demagnetization conditions could be detected with the $x, y$, or $z$ direction flux and with the Hall sensors at different locations. Measurement of the $z$ direction flux was selected in this paper, as it was shown to be relatively sensitive to the fault conditions while being relatively insensitive to the interference from the flux produced by the stator endwinding current [18]-[20].

If the flux inside the motor is measured with the analog Hall sensors at the given position, the leakage flux produced by the PMs can be measured directly with high precision [21]. Rotor eccentricity changes the relative distance between the Hall sensor and PM, since the Hall sensor is fixed to the stator, whereas the eccentric rotor rotates with respect to its own center of rotation. A 3D FE simulation of the Hall sensor measurement was performed as the rotor of Fig. 2(b) was moved between $y=-$ $0.5 \mathrm{~mm}$ and $+0.5 \mathrm{~mm}$ in the radial direction. This corresponds to $-62.5 \%$ to $+62.5 \%$ airgap eccentricity for the IPMSM with 0.8 $\mathrm{mm}$ airgap. The results of the peak value of the flux density in the $z$ direction plotted in Fig. 3(b) shows that it is very sensitive

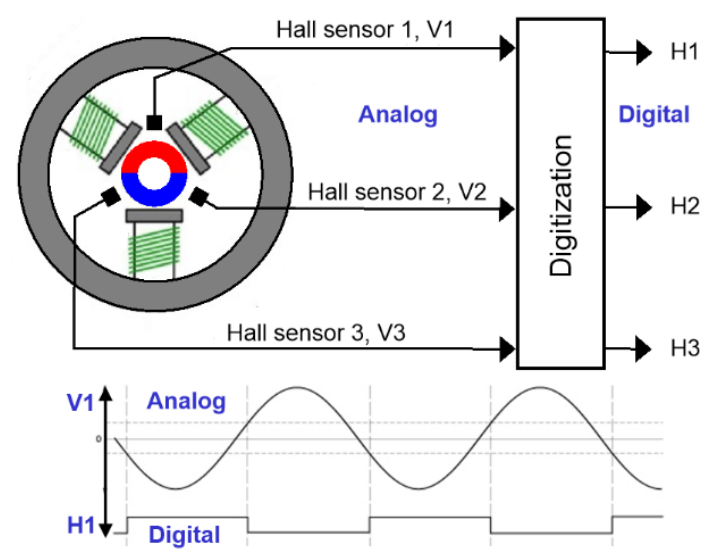

Fig. 1 Schematic of Hall effect field sensor analog measurement and digitization for obtaining rotor position

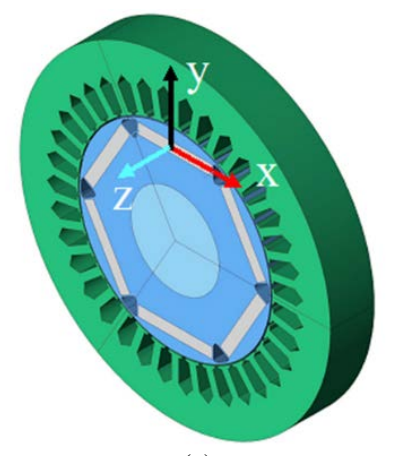

(a)

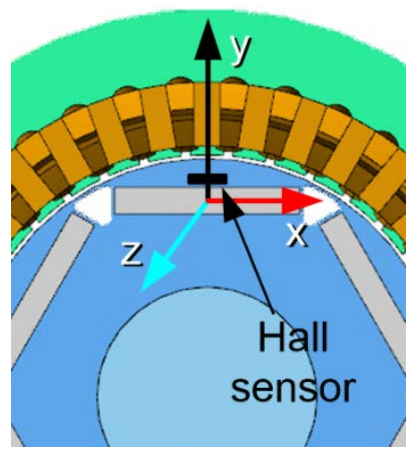

(b)
Fig. 2 3D FE model of $7.5 \mathrm{~kW}, 6$ pole IPMSM: (a) reference circumferential $(x)$, radial $(y)$ and axial $(z)$ directions; (b) location of Hall sensor installed for FE and experimental verification of proposed method. 
to and changes almost linearly with radial displacement of the $\mathrm{PM}$ rotor due to eccentricity. If the IPMSM rotor is in an ideal, healthy, and concentric condition, the radial distance between the Hall sensor and PMs does not change. The flux measurement is therefore a symmetric sinewave, as shown in the $3 \mathrm{D} \mathrm{FE}$ results of Fig. 4. If the motor is operating with dynamic eccentricity (DE), the radial distance between the PMs and fixed Hall sensor changes with rotor rotation, causing variation in the amplitude of the Hall sensor measurement. The Hall sensor therefore measures a distorted sinewave with asymmetric flux peaks, as shown in the FE simulation results of Fig. 4 with $30 \%$ DE [19], [21]. Although the FE results for 30\% DE are shown, it can be deduced from Fig. 3(b) that the change in the peaks of the Hall sensor measurements would be proportional to the severity of DE.

If the rotor PMs are not symmetrical due to local demagnetization or damage, the flux measurement of the Hall sensor is smaller when the defective PM passes the Hall sensor. The FE results of the Hall sensor measurement with one of the six PMs demagnetized by $5 \%$ is shown in Fig. 4. It can be seen that the flux waveform is distorted with the peak of the flux that corresponds to the demagnetized PM being lower. The peaks that correspond to the rest of the PMs do not change. Since DE and local demagnetization distort the Hall sensor measurement in a different way, the two types of rotor faults can be distinguished.

In [18]-[19], the influence of non-idealities such as manufacturing imperfections, PM temperature variation, stator endwinding current interference, and calibration/temperature dependency of Hall sensor characteristics are analyzed. Algorithms that are immune to the imperfections are also proposed for practical implementation. In addition, some alternative options for digital implementation are suggested for low cost drive systems [19]-[20]. The FE results show that Hall sensor based monitoring can provide sensitive and reliable detection of rotor eccentricity and local demagnetization by observing the change in the peaks of the flux measurements.

\section{B. Rotating Load Asymmetries}

Any type of mechanical defect or imperfection in the PMSM system that produces “once per revolution (or 1X)" vibration causes oscillation in the load torque at rotor speed given by $f_{r}$ in (1). There are a number of mechanical imperfections in the coupling or load that can produce $f_{r}$ components such as load unbalance, misalignment, eccentricity (load), mechanical looseness, mechanical wear, turbulence in flow, load fluctuation, etc [12]-[13]. Unlike PMSM rotor defects, these imperfections do not have any influence on the amplitude of the Hall sensor flux measurements, since the rotor MMF or airgap does not change with coupling or load defects.

\section{ANALYSIS OF MCSA-BASED DETECTION OF PMSM ROTOR AND LOAD DEFECTS}

MCSA can also be used to detect the presence of rotor eccentricity or local PM demagnetization in the motor, as demonstrated in [2]-[3], [7]-[11]. Eccentricity results in uneven airgap reluctance distribution, and local PM demagnetization causes the rotor MMF distribution to be asymmetrical, which both result in distortion of the airgap flux [22]-[23]. The distortion in the airgap flux induces $f_{r}$ component sidebands of $f_{s}$ in the stator current given by $f_{\text {fault }}$ in (2) that is observable with MCSA [2]-[3]. This is referred to as "amplitude modulation" since the MMF or airgap reluctance variation results in distortion in the amplitude of the airgap flux that influences the stator current. This component leads to an increase in the oscillating load torque components at $f_{r}$ that can be detected with vibration analysis [22]-[23].

MCSA can also provide indications of mechanical load defects listed in II.B since fluctuation in load torque at $f_{r}$ causes modulation of the stator current and produces the $f_{r}$ sidebands of $f_{s}$ in the current spectrum given by $f_{\text {fault }}$ in (2) [2]-[3], [22]-[24]. This is referred to as "phase modulation" since the fault

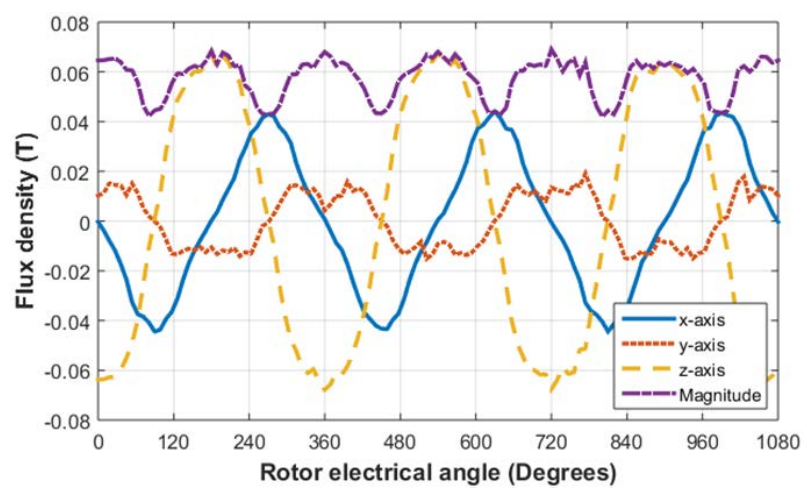

(a)

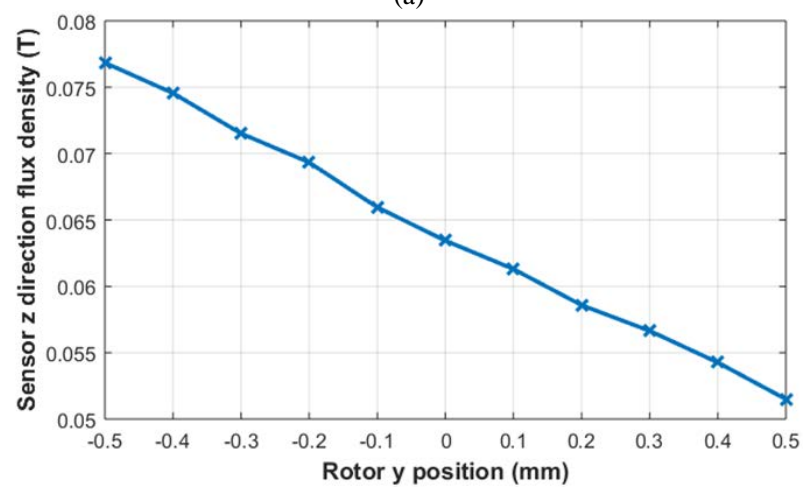

(b)

Fig. 3 (a) $x$-, $y$-, and $z$-axis flux density as a function of rotor electrical angle; (b) axial (z) direction peak flux measurement as a function of rotor radial $(y)$ location (corresponds to $-62.5 \%$ and $+62.5 \%$ rotor eccentricity)

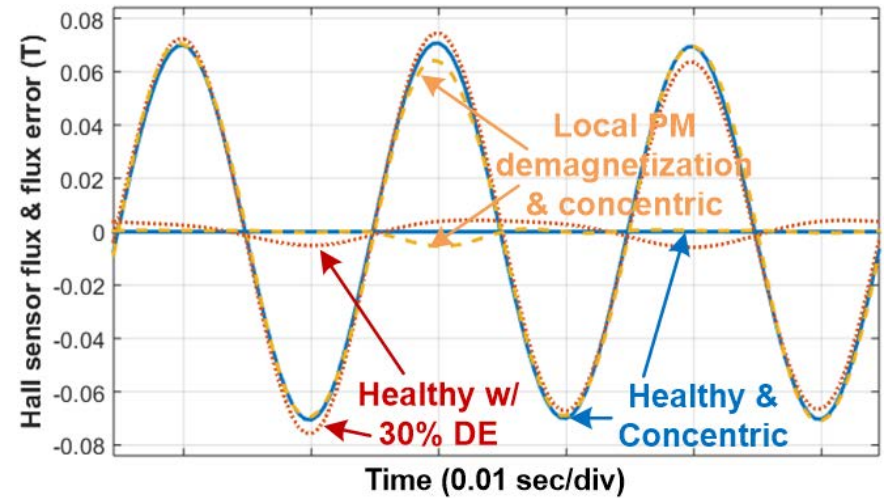

Fig. 4 3D FE simulation of Hall sensor flux measurement and error for cases of healthy concentric rotor; rotor with $30 \%$ dynamic eccentricity (DE); and local PM demagnetization in one magnet (motor speed: 1000 rpm) 
component is induced due to fluctuation in the angle of the airgap flux. There is always some degree of inherent imperfection in the coupling or load such as load unbalance, misalignment, or eccentricity despite the effort to minimize it during the manufacturing or commissioning stage [12]-[13]. Some loads such as compressors have inherent rotor position dependent oscillations that produce an $f_{r}$ component comparable or larger than that produced by the fault. These components are likely to increase during operation due to multiple reasons.

The $f_{\text {fault }}$ component is produced by PM rotor faults and load imperfections at an identical frequency in the stator current spectrum, and there is no known means of identifying the cause. It can provide indications on both types of faults, but it could lead to a serious problem of false indications when rotor and load faults co-exist, as will be shown in this paper. A simplified qualitative analysis is given to describe what is expected to occur when rotor and load defects are simultaneously present. For simplicity, only the first $(k=1)$ lower sideband component of $f_{\text {fault }}$ in (2) is considered, as it is the most prominent component used for condition monitoring. The stator current, $i_{s}$, can be expressed as the sum of the fundamental, and rotor- and load-induced $k=1$ lower sideband components as

$$
i_{s}=I_{s 1} \cos \left(2 \pi f_{s} t\right)+\left\{\begin{array}{l}
I_{s r} \cos \left(2 \pi f_{s} t-2 \pi f_{r} t-\emptyset_{r}\right) \\
+I_{s l} \cos \left(2 \pi f_{s} t-2 \pi f_{r} t-\emptyset_{l}\right)
\end{array}\right\},
$$

where subscripts $r, l$, and $s$ represent the rotor, load, and fundamental components, and $\phi$ represents the phase angle.

Induction of the $f_{r}$ sideband components in $i_{s}$ is a complicated phenomenon where the amplitude, $I_{s r}, I_{s l}$, and phase angle, $\phi_{r}, \phi_{l}$, depend on many factors such as system rotational inertia, electrical motor electrical parameters, the severity and angular position of the defects, etc [22]-[24]. Since the two types of defects are completely independent of each other and occur at a random angular position, the relative amplitude, $I_{s r} / I_{s l}$, and phase angles, $\phi_{r}-\phi_{l}$, between the rotor and load components are random and unknown. This makes separation of the two components very difficult. If rotor and load defects co-exist, the amplitude of the $f_{\text {fault }}$ component in the stator current, $I_{s, f s-f r}$, can be derived from (3) as

$$
I_{s, f_{s}-f_{r}}=\sqrt{I_{s r}^{2}+2 I_{s r} I_{s l} \cos \left(\emptyset_{r}-\emptyset_{l}\right)+I_{s l}^{2}} .
$$

This shows that the amplitude of the $f_{\text {fault }}$ component is a function of the amplitude and phase angle of the individual components produced by rotor and load defects. The amplitude of $I_{s, f s-f r}$ is calculated as a function of $I_{s r} / I_{s l}$ and $\phi_{r}-\phi_{l}$ from (3) and plotted in Fig. 5 to show how the $f_{\text {fault }}$ component can change due to interaction between the rotor and load defect induced components. Since the difference in phase angles is random, the two components can add or cancel and be anywhere in the shaded region depending on $\phi_{r}-\phi_{l}$ for a given value of $I_{s r} / I_{s l}$.

Considering that there is always some degree of inherent asymmetry in the PMSM rotor, coupling, or load, which can increase over time, these components can add to produce a false positive alarm. If the rotor and load defects co-exist and cancel each other out, a false negative alarm can be produced. False positive and negative MCSA indications causes MCSA to fail, and can have significant consequences in terms of economic loss. Interaction between the different $f_{\text {fault }}$ components degrades the reliability and sensitivity of MCSA, but there is currently no known means of separating the influence of rotor faults and load defects. The same problem of interaction between $f_{r}$ components produced by rotor and load defects is also present when vibration analysis is used.

\section{Advantages of Hall SENSOR BASED MONITORING}

Although the remote monitoring capability with existing current sensors makes MCSA an attractive option for detecting faults in PMSM systems, the limitations were identified as:

- Fault detectability dependent on the winding configuration and motor topology

- False indications due to interaction between rotor and load defects

- Requirement of steady state operation: complexity in timefrequency transformation techniques required for variable speed applications

The sensitivity and reliability of Hall sensor based detection of PMSM eccentricity and local PM demagnetization has been verified in previous work [19]-[20]. It is obvious that Hall sensor based detection of rotor faults is not influenced by the stator winding or motor topology since the asymmetry in the pattern of PM axial leakage flux is directly measured. In this work, it is shown that Hall sensor based detection of rotor faults is not influenced by the second and third limitations listed above for cases where MCSA fails.

\section{A. Classification of Rotor and Load Defects}

The underlying concept behind Hall sensor based classification of rotor and load defects proposed in this paper is very simple. Since analog Hall sensors provide direct measurement of the leakage flux inside the motor, it can reliably detect rotor eccentricity and local PM demagnetization by monitoring anomalies in the flux pattern, as described in II.A. However, any type of load torque oscillation produced by mechanical defects described in II.B does not cause distortion in the rotor MMF or airgap reluctance, and therefore, does not have any influence on the Hall sensor measurement of PM leakage flux. Therefore, change in the flux pattern of Hall sensor measurements indicates a fault in the PMSM rotor regardless of the MCSA indications. On the other hand, an increase in the $f_{\text {fault }}$ component in MCSA without change in the Hall sensor flux

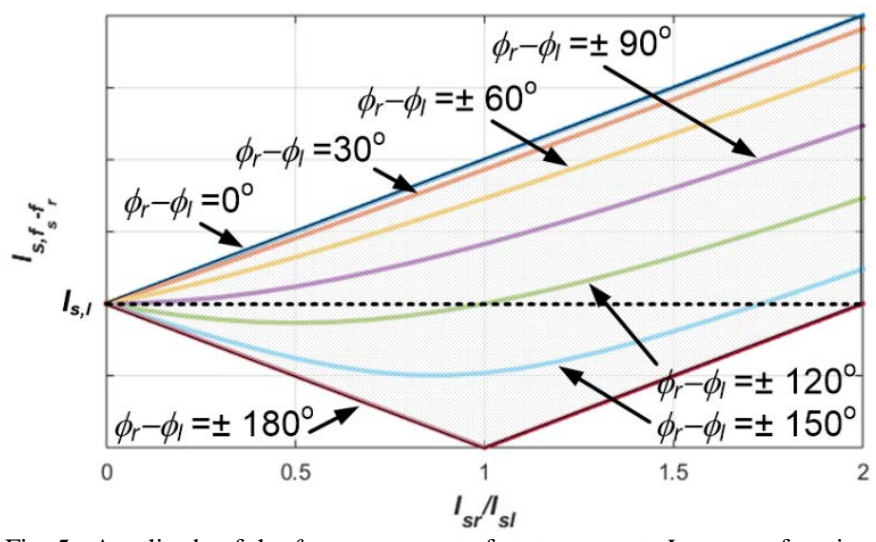

Fig. 5 Amplitude of the $f_{\text {fault }}$ component of stator current, $I_{s, f s-f r}$, as a function of the relative amplitude, $I_{s r} / I_{s l}$, and phase angle, $\phi_{r}-\phi_{l}$, of the individual rotor and load defect induced components 
pattern indicates that oscillations in the load torque is present due to a mechanical imperfection described in II.B. This allows the rotor faults and torque oscillations due to load defects to be distinguished. An example of a suitable application is when the PMSM is used for traction motors, since the mechanical stress is likely to cause rotor eccentricity and load defects.

There are no other known means of distinguishing the two types of faults other than off-line testing to the best of authors knowledge. In [4]-[6], measurement of radial or tangential flux on the stator frame or in the airgap is proposed for detection of PMSM faults. According to [25]-[26], the influence of stator current produced flux is prevalent in the airgap or frame flux measurements. Therefore, it is difficult to clearly distinguish the rotor and load related faults since the $f_{r}$ components present in the stator current are both reflected in the flux measurements, unlike the Hall sensors. If the PMSM system is equipped with analog Hall sensors at the location shown in Fig. 2, they are not sensitive to stator current interference, as demonstrated in [18][20]. Using the Hall sensor analog signal to complement MCSA to separate rotor and load defects can provide a significant advantage in terms of sensitivity and reliability considering that MCSA based fault detection can fail, as described in III.

\section{B. Detection of Rotor Faults under Transient Conditions}

There are many PMSM drive systems employed in the transportation, aerospace, or other industries that require continuously varying speed and/or load applications. Variation in the fundamental frequency, $f_{s}$, causes variation in the value of $f_{\text {fault, }}$ and tends to flatten the peaks if conventional MCSA is applied. Since MCSA is ineffective for many applications, a significant percentage of the work on PMSM fault detection is dedicated to detecting faults under non-stationary conditions where the frequency and amplitude of the stator current vary [9][11]. Monitoring of $f_{\text {fault }}$ components under non-stationary conditions requires time-frequency analysis techniques, which are computationally intensive. Implementation of such algorithms could be a limitation for many PMSM applications. When the PM flux is measured during non-stationary conditions, the amplitude of the flux measured by the Hall sensor does not change with rotor speed and is insensitive to stator current amplitude [18]. This is another important advantage of Hall sensor based rotor fault detection, since it can provide reliable detection of faults without heavy computational requirements. Rotor faults that produce nonuniform MMF or reluctance cause the Hall sensor output voltage peaks to fluctuate, whereas oscillation in the load torque due to load defects do not influence the Hall sensor output. Fluctuation in the flux peaks, which are not influenced by non-stationary operating conditions, can be monitored for reliable detection of faults, as will be demonstrated in V.B.

\section{EXPERIMENTAL STUDY}

\section{A. Experimental Test Setup}

An experimental study was performed on a $2.2 \mathrm{~kW}, 6$ pole IPMSM to verify the claims made on the advantages of the proposed Hall sensor based fault detection method. The end shields of the test machine were replaced with custom designed shields to include adjustable SE and DE sleeves, a Hall sensor, and a position encoder, as shown in Figs. 6-8. The original bearings of both ends were replaced with inner and outer sleeves installed on the new bearings (with smaller outer diameter (OD) and larger inner diameter (ID)) to fit between the rotor shaft and end shield, as shown in Figs. 6-7(a). The ID of the inner and outer sleeves were offset by $0 \%$ and $30 \%$ to produce DE and SE conditions. Two rotors were used for producing eccentricity (rotor A) and local demagnetization (rotor B) conditions. For producing local PM demagnetization/damage conditions, 3\% of one PM was chipped off from rotor B, as shown in Fig. 7(b). An aluminum disc was installed on the shaft of the motor, and a set of nuts and bolt was attached to the disc to emulate load unbalance conditions that produce $8.6 \%$ torque oscillations under rated load, as shown in Fig. 8. The location and weight of the bolt can be controlled to adjust the severity of rotating load

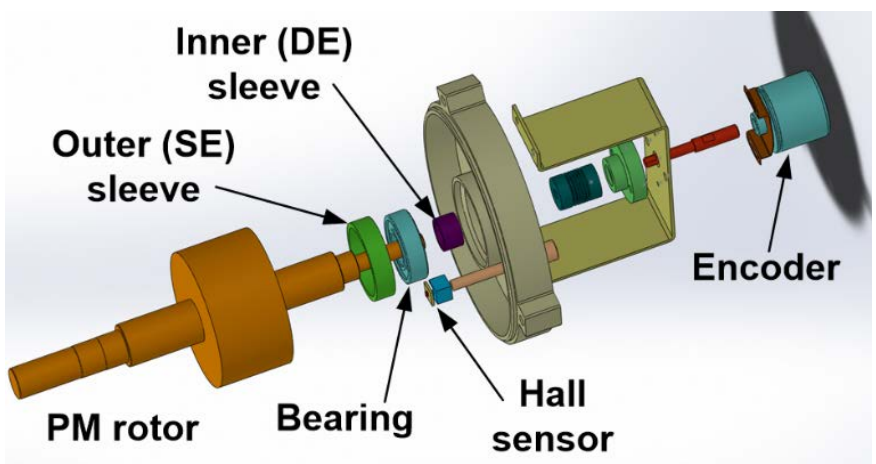

Fig. 6 Design drawing for custom-designed end shield for producing SE and DE conditions, and for installing Hall sensor and encoder

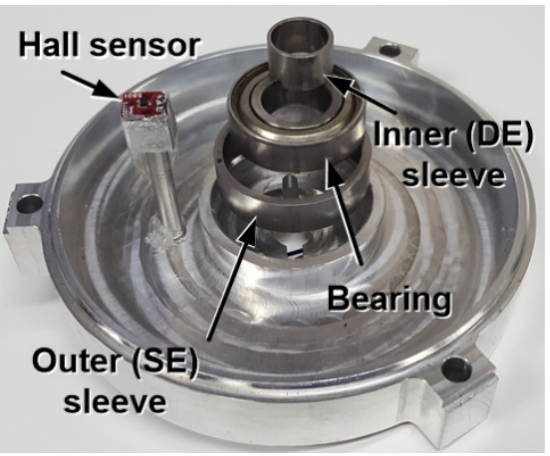

(a)

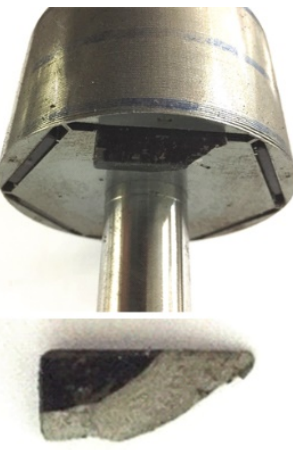

(b)
Fig. 7 (a) Photograph of custom designed end shield for introducing eccentricity conditions, Hall sensor, and encoder; (b) chipped PM for producing local PM demagnetization (rotor B)

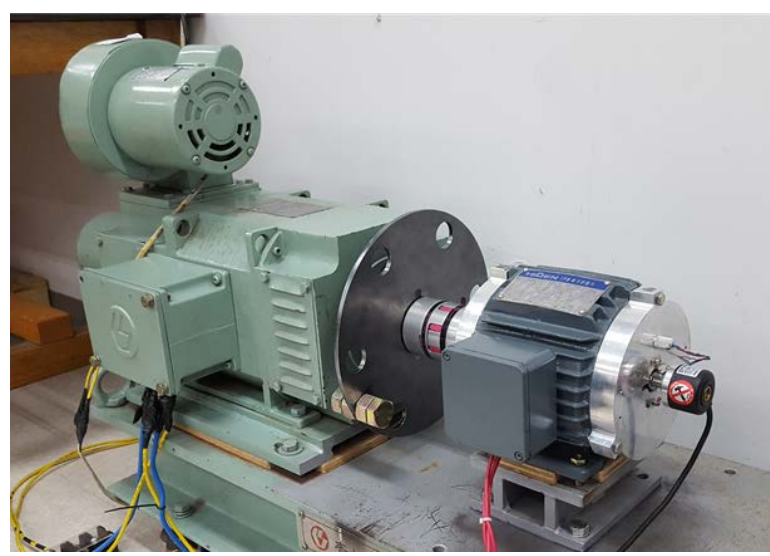

Fig. 8 Experimental setup of IPMSM and dc generator load, disc, and nuts/bolt for producing rotating load unbalance condition 
unbalance and how it interacts with the influence of rotor faults (local PM demagnetization).

Flux in the axial direction was measured with the Hall sensor attached to a rod protruding inward from the end shield, as shown in Figs. 6-7(a). The Hall sensor was positioned $3.2 \mathrm{~mm}$ apart from the PM in the $z$ direction and $3.5 \mathrm{~mm}$ above the center of the PM in the $y$ direction for measurement of the $z$-direction flux. A dc machine coupled to the test machine was operated as a generator supplying a resistor bank for controlling the load of the machine via field winding current. The Hall sensor and current data were acquired at $6.4 \mathrm{kHz}$ with the PMSM operated at $900 \mathrm{rpm}$ under rated load conditions.

\section{B. Experimental Results}

The flux measurement obtained from the analog Hall sensor over one mechanical rotation and current spectra obtained in steady state (30 secs) are shown in Figs. 9(a)-(b), respectively, under concentric and 30\% DE conditions (rotor A). It can be seen in Fig. 9(a) that there is distortion in the peaks of the flux measurements due to the eccentric rotor since the magnetic reluctance of the hall sensor flux path changes, as predicted in the FE simulation shown in Fig. 4. The pattern of the error between the flux of the eccentric and concentric rotor is consistent with the FE results. It is also clear from the MCSA results shown in Fig. 9(b) that the $f_{r}$ sideband component increases, as predicted in III. It should be noted that there is some degree of inherent SE condition in the motor that makes the DE condition a mixed eccentricity (ME) condition [27].

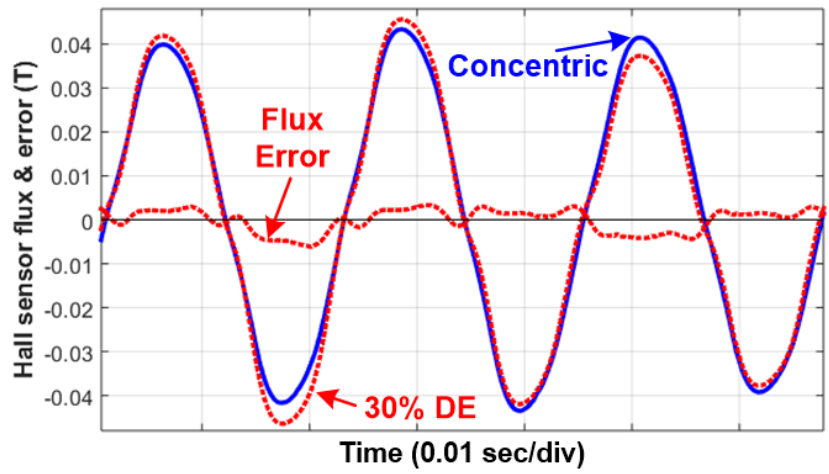

(a)

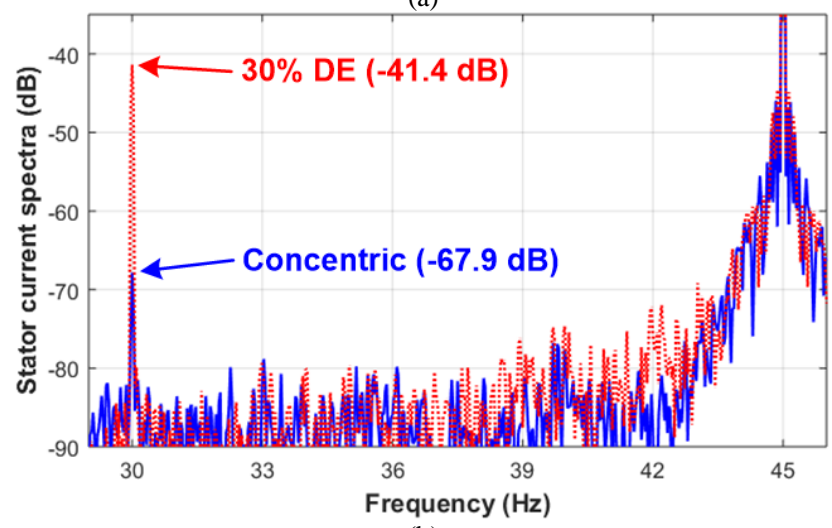

(b)

Fig. 9 Experimental measurements of (a) Hall sensor output signal and (b) MCSA results of $f_{r}$ sideband with concentric rotor and rotor with $30 \%$ $\mathrm{DE}$ and inherent SE (rotor A)
The Hall sensor measurement and MCSA results for the case of local PM demagnetization are shown in Figs. 10(a)-(b), respectively. The measurements were obtained before and after chipping off a piece of one PM from rotor B. It can be seen that one of the peaks of the Hall sensor measurements decreases due to the PM damage since the MMF of the rotor changes, as predicted by the FE simulation in Fig. 4. The lower $f_{r}$ sideband also increases with local demagnetization, as expected. It can be observed that the pattern of the Hall sensor measurement and the amplitude of the $f_{r}$ sideband in MCSA for a concentric, healthy case is different from that of Fig. 9(a) since a different rotor was used. The results shown in Figs. 9-10 confirm that faults in the PMSM rotor can be detected with both the Hall sensor measurements and MCSA, as predicted. The rotor faults can also be classified since the pattern of distortion in the flux measurements are different.

The Hall sensor and MCSA results for a rotor with $8.6 \%$ unbalanced load are shown in Figs. 11(a)-(b). The plots in Fig. 11(a) show that the Hall sensor measurements are not influenced by mechanical rotating unbalance in the load since there is no asymmetry in the MMF of magnetic reluctance of the Hall sensor flux path. However, Fig. 11(b) shows that torque oscillations produced with load unbalance leads to an increase in the $f_{r}$ sideband component when MCSA is performed. This shows that mechanical defects in the coupling or load that produce load oscillations influence MCSA, but not the peaks of the Hall sensor measurements, which allows rotor and load defects to be distinguished.

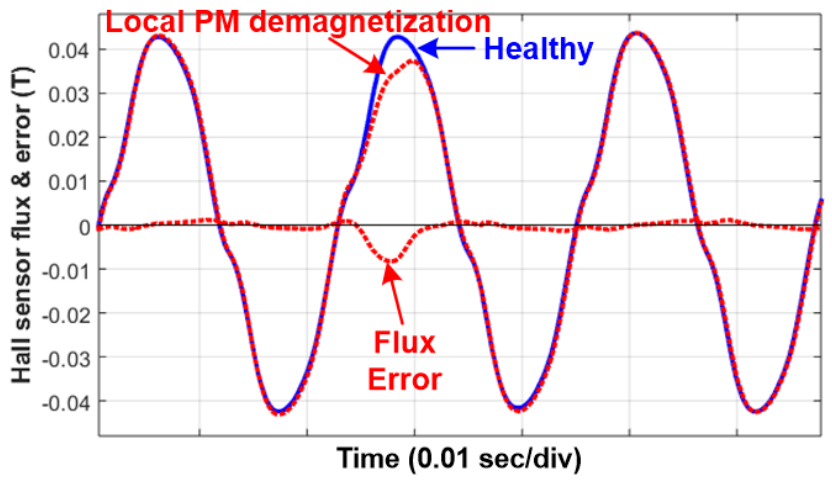

(a)

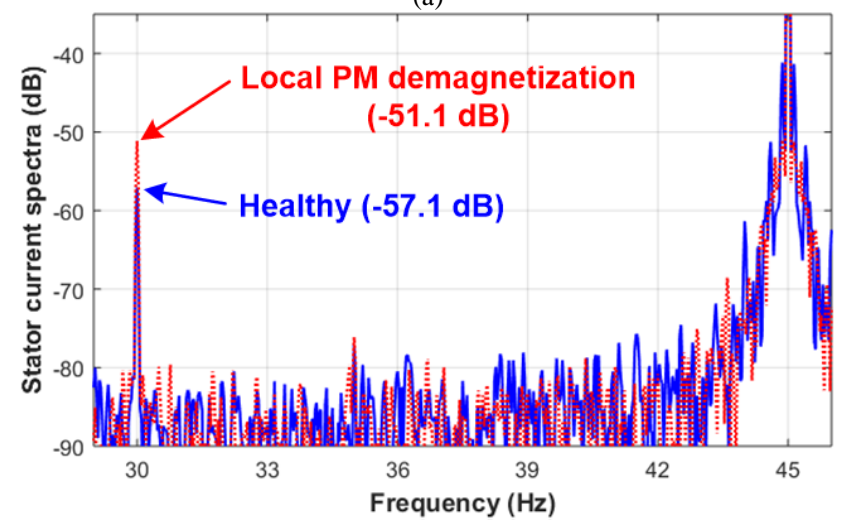

(b)

Fig. 10 Experimental measurements of (a) Hall sensor output signal and (b) MCSA results of $f_{r}$ sideband under healthy PMs and rotor with local demagnetization ( $3 \%$ of one PM chipped - rotor B) 
The Hall sensor measurements and MCSA results for the case where rotor and load related defects co-exist are shown in Fig. 12(a)-(b) to demonstrate how they can interact. The PMSM rotor with local PM demagnetization (rotor B) was operated without and with $8.6 \%$ load unbalance to show how the two types of defects interact. The location of the bolt was fixed at a position where the influence of local demagnetization and load unbalance are additive $\left(\phi_{r}-\phi_{l}=0^{\circ}\right)$, and then moved $180^{\circ}$ to a position where the two defects cancel each other out $\left(\phi_{r}-\phi_{l}=\right.$ $\left.180^{\circ}\right)$. It can be clearly seen that local demagnetization can be detected from both measurements if compared to the healthy case shown in Fig. 10. The MCSA results show that the $f_{r}$ sideband component can increase or decrease depending on the location of the load unbalance, as predicted in Fig. 5 of III. An important observation that can be made in Fig. 12(b) is that interaction of motor and load defects can cancel each other out resulting in a false negative MCSA indication (-66.0 dB is close to that of the healthy motor). Similarly, it can be deduced that minor defects in the motor and load that produce low $\mathrm{dB}$ levels can add to produce false positive MCSA indications. This also holds for vibration analysis, which is based on spectrum analysis. This implies that Hall sensor based detection of PMSM rotor faults can provide sensitive detection of PMSM rotor faults compared to MCSA since it is immune to load influence. This has not been reported in any existing paper in the literature, to the best of the authors' knowledge, and there currently is no known solution for identifying the cause of increase in $f_{r}$.

The Hall sensor measurement was obtained for the motor with local demagnetization (rotor B) under motor starting, which

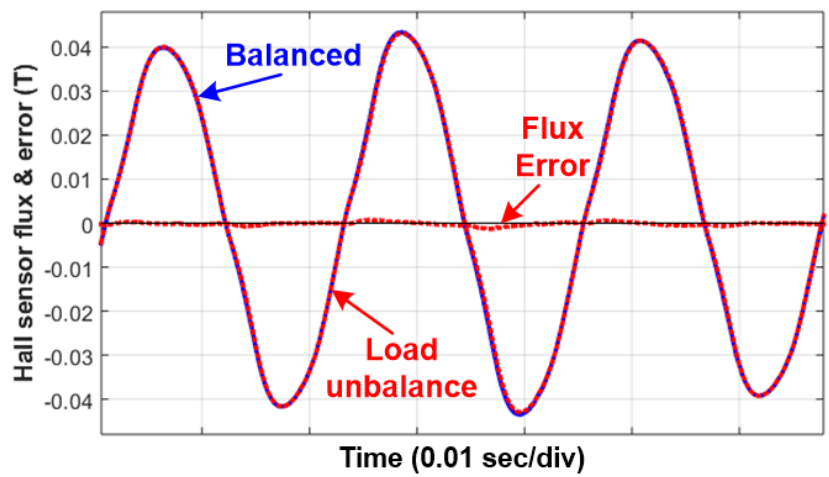

(a)

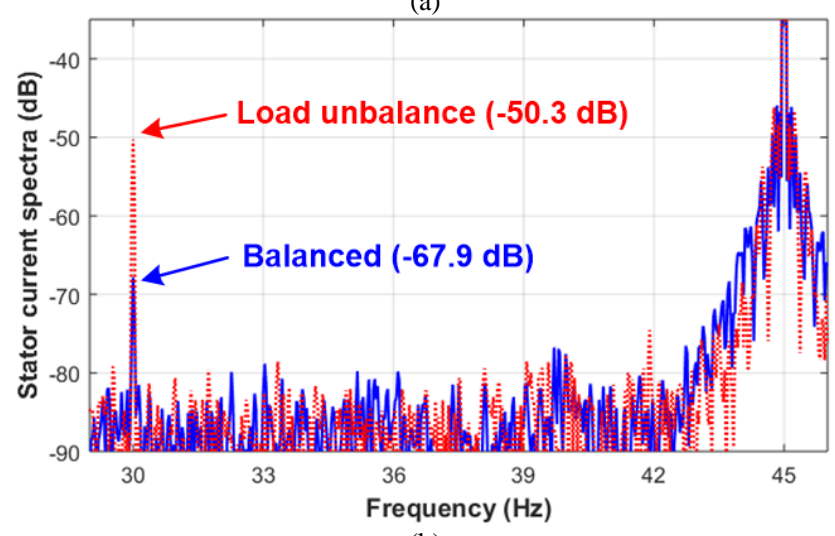

(b)

Fig. 11 Experimental measurements of (a) Hall sensor output signal and (b) MCSA results of $f_{r}$ sideband under load unbalance that results in $0 \%$ and $8.6 \%$ torque oscillations at $f_{r}$ (rotor A) is the most extreme non-stationary condition, to observe the detectability of rotor faults under transients. The Hall sensor, stator current, and rotor speed under motor acceleration from standstill to 900 rpm are shown in Figs. 13(a)-(c), respectively. The rotor speed was reconstructed from the $z$ signal output of the encoder in Fig. 13(c). It can be seen in Fig. 13(a) that there is a dip in one of the positive peaks per one rotor revolution due to the demagnetized PM. The other peaks produced by the healthy PMs are almost constant. The Hall sensor output voltage signal resembles that of the rotor with local demagnetization shown in Figs. 10(a) and 12(a) regardless of the abrupt change in the rotor speed. There is some current dependent fluctuation in the Hall sensor signal amplitude, but does not influence the detectability of the rotor fault since the relative peaks can be compared. This shows that rotor faults can be easily detected by observing the time domain signals without the requirement of a computationally intensive time-frequency transformation.

\section{CONCLUSION}

A method for detecting and classifying rotor and load defects in PMSM drive systems that utilizes the Hall sensor analog signals available for control was presented in this paper. It was shown that interaction between the signatures produced by rotor and load defects could cause false indications when conventional spectrum analysis based detection methods (MCSA) is applied. The main advantage of Hall sensor based fault detection is its capability of detecting and classifying rotor and load defects. Another advantage of the proposed method

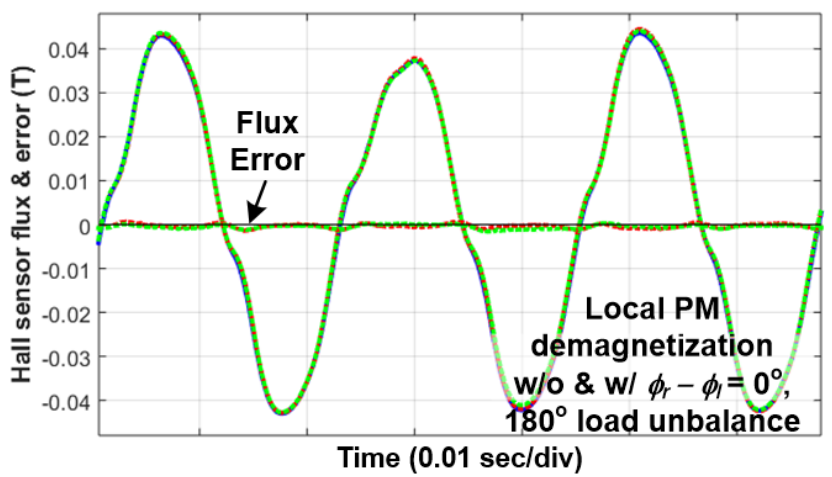

(a)

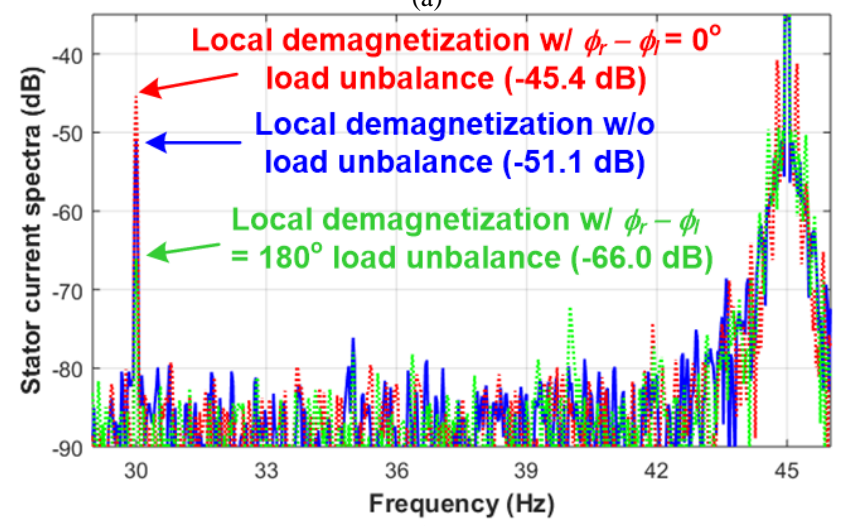

(b)

Fig. 12 Experimental measurements of (a) Hall sensor output signal and (b) MCSA results of $f_{r}$ sideband under local PM demagnetization (3\% of one PM chipped) and without and with load unbalance that results in $0 \%, 8.6 \%$ torque oscillations $\left(\phi_{r}-\phi_{l}=0^{\circ} \& 180^{\circ}\right)$ at $f_{r}$ (rotor B) 


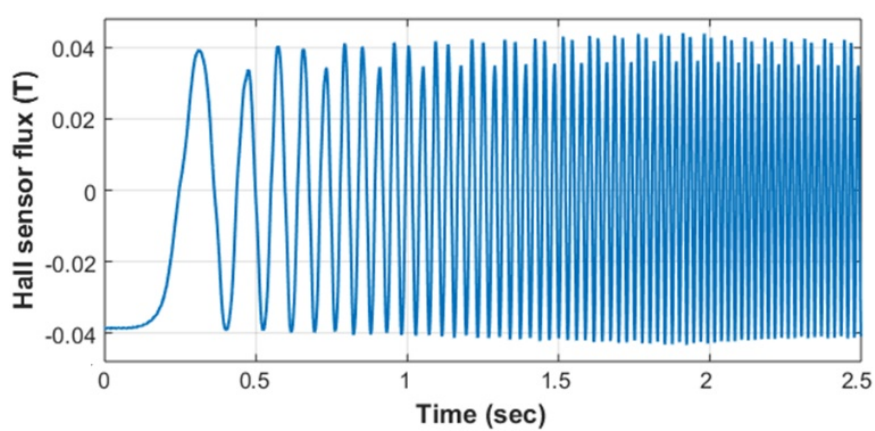

(a)

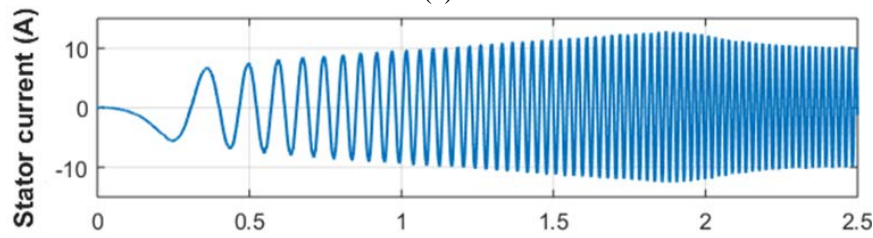

(b)

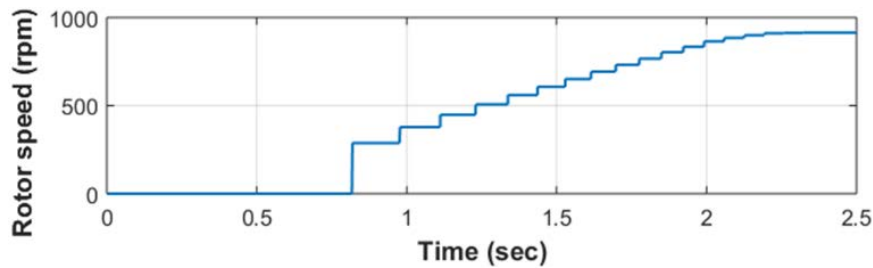

(c)

Fig. 13 Experimental measurements of (a) Hall sensor flux, (b) stator current, and (c) reconstructed rotor speed under acceleration of rotor (from z phase of encoder) under motor acceleration from standstill to $900 \mathrm{rpm}$

was identified as its simple and reliable detection capability under transient conditions for applications with speed variations.

The claims made in this paper were verified through experimental testing under local demagnetization, rotor eccentricity, and load unbalance conditions. It was shown that the proposed method can provide reliable separation of rotor and load defects for cases where they co-exist. It was also shown that rotor faults can be easily detected under motor transients. This is meaningful considering that conventional spectrum analysis methods based on current (MCSA) or vibration measurements fail under the two conditions. The proposed method is expected to provide sensitive and reliable detection and separation of rotor and load defects independent of motor model, design, or operating conditions at low added cost.

\section{ACKNOWLEDGEMENT}

This work was supported by the "Human Resources program in Energy Technology" of the Korea Institute of Energy Technology Evaluation and Planning(KETEP) granted financial resource from the Ministry of Trade, Industry \& Energy, Republic of Korea (No. 20174030201820)

\section{REFERENCES}

[1] J. Hong, et. al., "Detection and classification of rotor demagnetization and eccentricity faults for PM synchronous motors," IEEE Trans. Ind. Appl., vol. 48, no. 3, pp. 923-932, May/June 2012.

[2] W. le Roux, R.G. Harley, and T.G. Habetler, "Detecting rotor faults in low power permanent magnet synchronous machines," IEEE Trans. Power Electron., vol. 22, no. 1, pp. 322-328, Jan. 2007.
[3] S. Rajagopalan, et. al., "Dynamic eccentricity and demagnetized rotor magnet detection in bldc motors operating under different load conditions," IEEE Trans. Power Electron., vol. 22, no. 5, pp. 2061-2069, Sept. 2007.

[4] B.M. Ebrahimi, J. Faiz, "Magnetic field and vibration monitoring in permanent magnet synchronous motors under eccentricity fault," IET Elec. Power Appl., vol. 6, no. 1, pp. 35-45, January 2012.

[5] T. Goktas, M. Zafarani, K.W. Lee, B. Akin, T. Sculley, "Comprehensive analysis of magnet defect fault monitoring through leakage flux," IEEE Trans. Magn., vol. 53, no. 4, pp. 1-10, April 2017.

[6] W. Doorsamy, A.A.E. Abdallh, W.A. Cronje, L. Dupré, “An experimental design for static eccentricity detection in synchronous machines using a Cramér-Rao lower bound technique," IEEE Trans. Energy Convers., vol. 30, no. 1, pp. 254-261, March 2015.

[7] T. Goktas, M. Zafarani, B. Akin, "Discernment of broken magnet and static eccentricity faults in permanent magnet synchronous motors," IEEE Trans. Energy Convers., vol. 31, no. 2, pp. 578-587, June 2016.

[8] M. Zafarani, T. Goktas, B. Akin, S.E. Fedigan, "An Investigation of Motor Topology Impacts on Magnet Defect Fault Signatures,” IEEE Trans. Ind. Electron., vol. 64, no. 1, pp. 32-42, Jan. 2017.

[9] J. Rosero, J.L. Romeral, J. Cusido, J.A. Ortega, A. Garcia, "Fault detection of eccentricity and bearing damage by means of wavelet transforms decomposition of the stator current” Proc. of IEEE APEC, pp. 111-116, 2008.

[10] M. Delgado Prieto, et. al., "Feature extraction of demagnetization faults in PMSMs based on box-counting fractal dimension," IEEE Trans. on Ind. Electron., vol. 58, no. 5, pp. 1594-1605, May 2011.

[11] S. Rajagopalan, et. al., "Nonstationary motor fault detection using recent quadratic time-frequency representations,” IEEE Trans. on Ind. Appl., vol. 44, no. 3, pp. 735-744, May/June 2008.

[12] V. Wowk, Machinery Vibration. New York: McGraw-Hill, 1991.

[13] J.E. Berry, "Analysis III: introduction to special vibration diagnostic techniques and how to analysis low, high, and variable speed machines," Technical Associates of Charlotte, 1998.

[14] Faulhaber.com, "Brushless Dc servomotors," Series 22 BX4323268BX4, Mar. 2017. [Online]. Available: https://fmcc.faulhaber.com

[15] Technosoft.com, "Drive solutions for motion control applications product overview,", Mar. 2017. [Online]. Available: http://www.technosoftmotion.com/en/products

[16] Y. F. Shi, Z. Q. Zhu, D. Howe, "EKF-based hybrid controller for PM brushless motors combining hall sensors and a flux-observer-based sensorless technique," Proc. of IEEE IEMDC, pp. 1466-1472, May 2005.

[17] X. Song, J. Fang, B. Han, "High-precision rotor position detection for high-speed surface PMSM drive based on linear hall-effect sensors," IEEE Trans. Power Electron., vol. 31, no. 7, pp. 4720 -4731, July 2016.

[18] D. Fernandez et al., "Permanent Magnet Temperature Estimation in PM Synchronous Motors Using Low-Cost Hall Effect Sensors," IEEE Trans. on Ind. Appl., vol. 53, no. 5, pp. 4515-4525, Sept./Oct. 2017.

[19] Y. Park, et al., "On-line detection of rotor eccentricity for PMSMs based on hall-effect field sensor measurements," Proc. of IEEE ECCE, pp. 4678-4685, 2017.

[20] D. Diaz, D. Fernandez, Y. Park, A. B. Diez, S. B. Lee and F. Briz, "Detection of demagnetization in permanent magnet synchronous machines using hall-effect sensors," IEEE Trans. Ind. Appl., vol. 54, no. 4, pp. 3338-3349, July/August 2018.

[21] Y. Park, C. Yang, S.B. Lee, D. Lee, D. Fernandez, D. Reigosa, and F. Briz, "On-line detection and classification of rotor and load defects in PMSMs based on hall sensor measurements," Proc. of ECCE, Sept. 2018.

[22] M. Blodt et al., "Distinguishing Load Torque Oscillations and Eccentricity Faults in Induction Motors Using Stator Current Wigner Distributions,” IEEE Trans. Ind. Appl., vol. 45, no. 6, pp. 1991-2000, Nov./Dec. 2009.

[23] M. Blodt, P. Granjon, B. Raison, G. Rostaing, "Models for Bearing Damage Detection in Induction Motors Using Stator Current Monitoring," IEEE Trans. Ind. Electron., vol. 55, no. 4, pp. 1813-1822, April 2008.

[24] F. Immovilli, A. Bellini, R. Rubini, C. Tassoni, "Diagnosis of Bearing Faults in Induction Machines by Vibration or Current Signals: A Critical 
Comparison,” IEEE Trans. Ind. Appl., vol. 46, no. 4, pp. 1350-1359, July/Aug. 2010.

[25] H. Henao, C. Demian, G.A. Capolino, "A frequency-domain detection of stator winding faults in induction machines using an external flux sensor," IEEE Trans. Ind. Appl., vol. 39, no. 5, pp. 1272-1279, Sept.-Oct. 2003.
[26] Bellini, C. Concari, G. Franceschini, C. Tassoni, A. Toscani, "Vibrations, currents and stray flux signals to asses induction motors rotor conditions," Proc. of IEEE IECON, pp. 4963-4968, 2006.

[27] D.G. Dorrell, W.T. Thomson, S. Roach, "Analysis of airgap flux, current, and vibration signals as a function of the combination of static and dynamic airgap eccentricity in 3-phase induction motors," IEEE Trans. Ind. Appl., vol. 33, no. 1, pp. 24-34, Jan/Feb 1997. 